\title{
Flux synthesis of a metal carbide hydride using anthracene as a reactant
}

Tate O. Engstrand, Emily M. Cope, Guillermo Vasquez, Jo W. Haddock, Mary B. Hertz, Xiaoping Wang*, and Susan E. Latturner*

Department of Chemistry and Biochemistry, Florida State University, Tallahassee, FL 32306

Neutron Scattering Division, Oak Ridge National Laboratory, Oak Ridge, Tennessee 37831

Corresponding author: slatturner@fsu.edu

\section{Supporting Information}


Table S1. List of atomic coordinates and thermal parameters for $\mathrm{La}_{15}\left(\mathrm{FeC}_{6}\right)_{4} \mathrm{H}$ in space group $\mathrm{P}$ 6 , determined from single crystal neutron diffraction data.

\begin{tabular}{|c|c|c|c|c|c|c|}
\hline Atom & Wyckoff site & $\mathbf{x}$ & $\mathbf{y}$ & $\mathbf{z}$ & $\mathbf{O c c}$ & $\mathbf{U}_{\text {eq }}$ \\
\hline $\mathrm{La} 1$ & $3 \mathrm{k}$ & $0.7511(1)$ & $0.0146(1)$ & $1 / 2$ & 1 & $0.0023(1)$ \\
\hline $\mathrm{La} 2$ & $1 \mathrm{~d}$ & $1 / 3$ & $2 / 3$ & $1 / 2$ & 1 & $0.0029(2)$ \\
\hline $\mathrm{La} 3$ & $3 \mathrm{j}$ & $0.0592(1)$ & $0.6678(1)$ & 0 & 1 & $0.0019(1)$ \\
\hline $\mathrm{La} 4$ & $2 \mathrm{~g}$ & 0 & 0 & $0.16175(8)$ & 1 & $0.0027(1)$ \\
\hline $\mathrm{La} 5$ & 61 & $0.94218(6)$ & $0.35040(7)$ & $0.25139(5)$ & 1 & $0.00201(7)$ \\
\hline $\mathrm{Fe} 1$ & $1 \mathrm{e}$ & $2 / 3$ & $1 / 3$ & 0 & 1 & $0.0019(2)$ \\
\hline $\mathrm{Fe} 2$ & $2 \mathrm{~h}$ & $1 / 3$ & $2 / 3$ & $0.22987(7)$ & 1 & $0.0020(1)$ \\
\hline $\mathrm{Fe} 3$ & $1 \mathrm{f}$ & $2 / 3$ & $1 / 3$ & $1 / 2$ & 1 & $0.0018(2)$ \\
\hline $\mathrm{C} 1$ & $3 \mathrm{j}$ & $0.8532(1)$ & $0.2925(1)$ & 0 & 1 & $0.0034(1)$ \\
\hline $\mathrm{C} 2$ & $3 \mathrm{j}$ & $0.9634(1)$ & $0.2288(1)$ & 0 & 1 & $0.0052(1)$ \\
\hline $\mathrm{C} 3$ & 61 & $0.14819(9)$ & $-0.29447(9)$ & $0.24970(6)$ & 1 & $0.00329(9)$ \\
\hline $\mathrm{C} 4$ & 61 & $0.00478(9)$ & $-0.2802(1)$ & $0.24763(6)$ & 1 & $0.00455(9)$ \\
\hline $\mathrm{C} 5$ & $3 \mathrm{k}$ & $0.8934(1)$ & $0.3761(1)$ & $1 / 2$ & 1 & $0.0034(1)$ \\
\hline $\mathrm{C} 6$ & $3 \mathrm{k}$ & $0.6469(1)$ & $-0.3202(1)$ & $1 / 2$ & 1 & $0.0045(1)$ \\
\hline $\mathrm{H} 1$ & $2 \mathrm{~g}$ & 0 & 0 & $0.408(1)$ & 0.5 & $0.053(3)$ \\
\hline
\end{tabular}

Table S2. List of atomic coordinates for $\mathrm{La}_{15}\left(\mathrm{FeC}_{6}\right)_{4} \mathrm{H}$ modelled in space group $\mathrm{P} 3$ for electronic structure calculation. All site occupancies 100\%.

\begin{tabular}{|c|c|c|c|c|}
\hline Atom & Wyckoff site & $\mathbf{x}$ & $\mathbf{y}$ & $\mathbf{Z}$ \\
\hline Lal & $3 \mathrm{~d}$ & $0.7511(1)$ & $0.0146(1)$ & $1 / 2$ \\
\hline $\mathrm{La} 2$ & $1 \mathrm{~b}$ & $1 / 3$ & $2 / 3$ & $1 / 2$ \\
\hline La3 & $3 \mathrm{~d}$ & $0.0592(1)$ & $0.6678(1)$ & 0 \\
\hline La4 & $1 \mathrm{a}$ & 0 & 0 & $0.16175(8)$ \\
\hline La4A & $1 \mathrm{a}$ & 0 & 0 & -0.16175 \\
\hline La5 & $3 \mathrm{~d}$ & $0.94218(6)$ & $0.35040(7)$ & $0.25139(5)$ \\
\hline La5A & $3 \mathrm{~d}$ & $0.94218(6)$ & $0.35040(7)$ & $-0.25139(5)$ \\
\hline $\mathrm{Fe} 1$ & $1 \mathrm{c}$ & $2 / 3$ & $1 / 3$ & 0 \\
\hline $\mathrm{Fe} 2$ & $1 b$ & $1 / 3$ & $2 / 3$ & $0.22987(7)$ \\
\hline $\mathrm{Fe} 2 \mathrm{~A}$ & $1 \mathrm{~b}$ & $1 / 3$ & $2 / 3$ & -0.22987 \\
\hline Fe3 & $1 \mathrm{c}$ & $2 / 3$ & $1 / 3$ & $1 / 2$ \\
\hline $\mathrm{C} 1$ & $3 \mathrm{~d}$ & $0.8532(1)$ & $0.2925(1)$ & 0 \\
\hline $\mathrm{C} 2$ & $3 \mathrm{~d}$ & $0.9634(1)$ & $0.2288(1)$ & 0 \\
\hline $\mathrm{C} 3$ & $3 \mathrm{~d}$ & $0.14819(9)$ & $-0.29447(9)$ & $0.24970(6)$ \\
\hline $\mathrm{C} 3 \mathrm{~A}$ & $3 d$ & $0.14819(9)$ & $-0.29447(9)$ & $-0.24970(6)$ \\
\hline $\mathrm{C} 4$ & $3 \mathrm{~d}$ & $0.00478(9)$ & $-0.2802(1)$ & $0.24763(6)$ \\
\hline $\mathrm{C} 4 \mathrm{~A}$ & $3 d$ & $0.00478(9)$ & $-0.2802(1)$ & $-0.24763(6)$ \\
\hline $\mathrm{C} 5$ & $3 \mathrm{~d}$ & $0.8934(1)$ & $0.3761(1)$ & $1 / 2$ \\
\hline C6 & $3 \mathrm{~d}$ & $0.6469(1)$ & $-0.3202(1)$ & $1 / 2$ \\
\hline H1 & $1 \mathrm{a}$ & 0 & 0 & $0.408(1)$ \\
\hline$H 1 A$ & $1 a$ & 0 & 0 & -0.408 \\
\hline
\end{tabular}

The hydride $2 g$ site in P-6 splits into two $1 a$ sites in P3. Half-occupancy of the $2 g$ site (P- 6$)$ is modelled as full occupancy of one of the $1 a$ sites in the P 3 model; other $1 a$ site (grey font) is unoccupied. 
Table S3. Selected interatomic distances in the structure $\mathrm{La}_{15}\left(\mathrm{FeC}_{6}\right)_{4} \mathrm{H}$

\begin{tabular}{|l|l|}
\hline Bond & Distance $(\AA)$ \\
\hline $\mathrm{Fe}(1)-\mathrm{C}(1)$ & $1.843(1)$ \\
\hline $\mathrm{Fe}(1)-\mathrm{La}$ & $3.2204(8)-3.5764(5)$ \\
\hline $\mathrm{Fe}(2)-\mathrm{C}(3)$ & $1.8320(7)$ \\
\hline $\mathrm{Fe}(2)-\mathrm{La}$ & $3.1645(5)-3.4500(8)$ \\
\hline $\mathrm{Fe}(3)-\mathrm{C}(5)$ & $1.831(1)$ \\
\hline $\mathrm{Fe}(3)-\mathrm{La}$ & $3.2323(8)-3.5539(5)$ \\
\hline $\mathrm{C}(1)-\mathrm{C}(2)$ & $1.338(1)$ \\
\hline $\mathrm{C}(3)-\mathrm{C}(4)$ & $1.3261(9)$ \\
\hline $\mathrm{C}(5)-\mathrm{C}(6)$ & $1.337(1)$ \\
\hline $\mathrm{C}-\mathrm{La}$ & $2.6458(8)-3.1183(8)$ \\
\hline $\mathrm{H}(1)-\mathrm{La}$ & $2.456(6)-2.65(1)$ \\
\hline
\end{tabular}

